ANNALES

POLONICI MATHEMATICI

$92.2(2007)$

\title{
Definable stratification satisfying the Whitney property with exponent 1
}

\author{
by Beata Kocel-Cynk (Kraków)
}

\begin{abstract}
We prove that for a finite collection of sets $A_{1}, \ldots, A_{s} \subset \mathbb{R}^{k+n}$ definable in an o-minimal structure there exists a compatible definable stratification such that for any stratum the fibers of its projection onto $\mathbb{R}^{k}$ satisfy the Whitney property with exponent 1.
\end{abstract}

Introduction. K. Kurdyka proved (in [4]) that for any locally finite family of subanalytic sets in $\mathbb{R}^{n}$ there exists a subanalytic stratification of $\mathbb{R}^{n}$ compatible with every element of the family and such that all strata satisfy the Whitney property with exponent 1 . The aim of our note is to prove a version with parameter of the above theorem for an o-minimal structure on $(\mathbb{R},+, \cdot)$.

THEOREM 1. Let $\mathcal{S}$ be an o-minimal structure on $(\mathbb{R},+, \cdot)$ and let $A_{1}, \ldots, A_{s} \subset \mathbb{R}^{k+n}$ be definable sets in $\mathcal{S}$. Then there exists a finite definable stratification of $\mathbb{R}^{k+n}$ compatible with the sets $A_{1}, \ldots, A_{s}$ and such that for any stratum $Q$ of this stratification and any point $y \in \pi(Q)$ the fiber $Q_{y}$ is (in some coordinate system in $\mathbb{R}^{n}$ ) a definable cell satisfying the Whitney property with exponent 1 (and coefficient depending only on $n$ ).

In the proofs we shall use properties of the closure of a definable cell and extensions of definable functions to the boundary.

1. Basic properties of o-minimal structures. In this section we collect some basic properties of o-minimal structures on $(\mathbb{R},+, \cdot)$, crucial for further considerations. Let us start with some definitions.

Definition 1 ([2]). A structure $\mathcal{S}$ on $\mathbb{R}$ consists of a collection $\mathcal{S}_{n}$ of subsets of $\mathbb{R}^{n}$, for each $n \in \mathbb{N}$, such that

(1) $\mathcal{S}_{n}$ is a boolean algebra of subsets of $\mathbb{R}^{n}$,

2000 Mathematics Subject Classification: 14P15, 32B20.

Key words and phrases: Whitney property, definable sets, o-minimal structure. 
(2) $\mathcal{S}_{n}$ contains the diagonals $\left\{\left(x_{1}, \ldots, x_{n}\right) \in \mathbb{R}^{n}: x_{i}=x_{j}\right\}$ for $1 \leq i<$ $j \leq n$,

(3) if $A \in \mathcal{S}_{n}$, then $A \times \mathbb{R}$ and $\mathbb{R} \times A$ belong to $\mathcal{S}_{n+1}$,

(4) if $A \in \mathcal{S}_{n+1}$, then $\pi(A) \in \mathcal{S}_{n}$, where $\pi: \mathbb{R}^{n+1} \rightarrow \mathbb{R}^{n}$ is the projection on the first $n$ coordinates.

We say that a set $A \subset \mathbb{R}^{n}$ is definable iff $A \in \mathcal{S}_{n}$. A function $f: A \rightarrow \mathbb{R}^{m}$ with $A \subset \mathbb{R}^{n}$ is called definable iff its graph is definable.

Definition 2 ([2]). A structure $\mathcal{S}$ on $\mathbb{R}$ is o-minimal iff

(1) $\{(x, y): x<y\} \in \mathcal{S}_{2}$ and $\{a\} \in \mathcal{S}_{1}$ for each $a \in \mathbb{R}$,

(2) each set in $\mathcal{S}_{1}$ is a finite union of intervals $(a, b),-\infty \leq a<b \leq+\infty$, and points $\{a\}$.

A structure on $(\mathbb{R},+, \cdot)$ is a structure on $\mathbb{R}$ containing the graphs of both addition and multiplication.

\section{Cell decomposition and stratification}

Definition 3 ([1]). Cells in $\mathbb{R}^{n}$ are definable sets defined in the following inductive way:

(1) The cells in $\mathbb{R}^{1}$ are exactly points and open intervals,

(2) Let $C \subset \mathbb{R}^{n}$ be a cell and let $f, g: C \rightarrow \mathbb{R}$ be continuous definable functions such that $f<g$ on $C$. Then

$$
(f, g):=\{(x, r) \in C \times \mathbb{R}: f(x)<r<g(x)\}
$$

is a cell in $\mathbb{R}^{n+1}$. Also, given a continuous definable function $f: C \rightarrow$ $\mathbb{R}$ on a cell $C$ in $\mathbb{R}^{n}$, the graph

$$
\Gamma(f)=\{(x, r) \in C \times \mathbb{R}: r=f(x)\}
$$

and the sets

$$
\{(x, r) \in C \times \mathbb{R}: f(x)<r\},\{(x, r) \in C \times \mathbb{R}: r<f(x)\}, C \times \mathbb{R}
$$

are cells in $\mathbb{R}^{n+1}$.

Definition 4 ([1]). A cell decomposition of $\mathbb{R}^{n}$ is a partition of $\mathbb{R}^{n}$ into finitely many cells defined in the following inductive way:

(1) A decomposition of $\mathbb{R}^{1}$ is a collection of open intervals and points of the following form:

$$
\left\{\left(-\infty, a_{1}\right),\left(a_{1}, a_{2}\right), \ldots,\left(a_{k},+\infty\right),\left\{a_{1}\right\}, \ldots,\left\{a_{k}\right\}\right\} .
$$

(2) A decomposition of $\mathbb{R}^{n+1}$ is a finite partition of $\mathbb{R}^{n+1}$ into cells $A$ such that the set of projections $\pi(A)$ is a decomposition of $\mathbb{R}^{n}$, where $\pi: \mathbb{R}^{n+1} \rightarrow \mathbb{R}^{n}$ is the projection on the first $n$ coordinates. 
In a similar way we define a $\mathcal{C}^{k}$ cell and $\mathcal{C}^{k}$ cell decomposition, by requiring that the functions in part (2) of Definition 3 are $\mathcal{C}^{k}$ functions.

Proposition $2([2])$. Any o-minimal structure $\mathcal{S}$ on $(\mathbb{R},+,$.$) admits \mathcal{C}^{1}$ cell decompositions, i.e.:

(1) If $A_{1}, \ldots, A_{k} \subset \mathbb{R}^{n}$ are definable sets then there exists a $\mathcal{C}^{1}$ cell decomposition of $\mathbb{R}^{n}$ compatible with $A_{1}, \ldots, A_{k}$.

(2) For each definable function $f: A \rightarrow \mathbb{R}$ with $A \subset \mathbb{R}^{n}$ there exists a cell decomposition of $\mathbb{R}^{n}$ partitioning $A$ and such that for every $C \subset A$ in the decomposition the restriction $f \mid C: C \rightarrow \mathbb{R}$ is a $\mathcal{C}^{1}$ function.

REMARK 3. Every o-minimal structure on $(\mathbb{R},+, \cdot)$ admits $\mathcal{C}^{k}$ cell decompositions (for any positive integer $k$ ), i.e. the above proposition holds with $\mathcal{C}^{1}$ replaced by $\mathcal{C}^{k}$.

Definition 5 . We call a definable subset of $\mathbb{R}^{n}$ which is a $\mathcal{C}^{k}$ submanifold of $\mathbb{R}^{n}$ a definable $\mathcal{C}^{k}$ stratum in $\mathbb{R}^{n}$.

A definable $\mathcal{C}^{k}$ stratification of $\mathbb{R}^{n}$ is a finite partition of $\mathbb{R}^{n}$ into definable $\mathcal{C}^{k}$ strata satisfying the following boundary condition: for any two strata $S, T$ of the partition, if $S \cap \partial T \neq \emptyset$ then $S \subset \partial T$.

Definition 6. A set $T$ definable in an o-minimal structure satisfies the Whitney property with exponent $\alpha$ (cf. [5]) if there exists a positive constant $C$ such that any points $p$ and $q$ in $T$ can be joined by a definable curve $\gamma$ with length $(\gamma) \leq C|p-q|^{\alpha}$.

\section{Angle between linear subspaces}

Definition 7. The angle between a linear subspace $X$ and a line $P$ in $\mathbb{R}^{n}$ is the number

$$
\delta(P, X)=\inf \{\sin (P, S): S \text { a line in } X\}
$$

where $\sin (P, S)$ denotes the sine of the angle between the lines $P$ and $S$.

The angle between linear subspaces $X$ and $Y$ in $\mathbb{R}^{n}$ is the number

$$
\delta(Y, X):=\sup \{\delta(P, X): P \text { a line in } Y\} .
$$

If $Y=0$ we put $\delta(0, X)=0$.

REMARK 4 ([4]).

(1) If $\operatorname{dim} X=\operatorname{dim} Y$ then $\delta(X, Y)=\delta(Y, X)$.

(2) If $\operatorname{dim} X \leq \operatorname{dim} Y \leq \operatorname{dim} Z$ then $\delta(Z, X) \leq \delta(Z, Y)+\delta(Y, X)$.

(3) Let $\mathbb{G}(k, m)$ be the Grassmannian of $k$-dimensional subspaces in $\mathbb{R}^{m}$. The mapping $\mathbb{G}(k, m) \times \mathbb{G}(k, m) \ni(X, Y) \mapsto \delta(X, Y) \in \mathbb{R}$ is continuous and semialgebraic. 
(4) For any $\alpha>0$ there exists $M>0$ such that if $\delta(P, X)>\alpha$ for some linear hyperplane $X$ and a line $P$ then $X$ is the graph of a linear map $\phi: P^{\perp} \rightarrow P$ satisfying $\|\phi\| \leq M$.

Lemma 5 ([4, Lem. 3]). For any nonnegative integers $r, n$ there exist $\varepsilon, m>0$ such that for any hyperplanes $X_{1}, \ldots, X_{r}$ in $\mathbb{R}^{n}$ there exists a line $P$ such that for any hyperplanes $Y_{1}, \ldots, Y_{r}$ satisfying $\delta\left(X_{i}, Y_{i}\right)<\varepsilon$ we have $\delta\left(P, Y_{i}\right)>m$.

4. Closure of a cell. The closure of a definable cell is also definable. In this section we shall give a description of the closure of a cell. We shall consider separately cells of graph and band types.

EXAmple 6. Consider the following cell of graph type in $\mathbb{R}^{3}$ :

$$
Q=\{(x, y, z): 0<x<1,0<y<1, z=x / y\} .
$$

The closure $\bar{Q}$ of $Q$ is not a graph, its fiber over any point from the closure of the projection of $\overline{\pi(Q)}=[0,1]^{2}$ different from $(0,0)$ consists of one point, whereas the fiber over $(0,0)$ is the half-line $[0, \infty)$.

We shall show that for any cell of graph type the set of points over which the fiber of the closure is infinite has small dimension.

Lemma 7. Let $f: Q \rightarrow \mathbb{R}$ be a continuous definable function defined on a cell of dimension $d$ in $\mathbb{R}^{n}$. There is a definable set $Z \subset \partial Q$ of dimension $\leq d-2$ such that $f$ has a continuous extension to $\bar{Q} \backslash Z$.

Proof. Let $Z:=\left\{x \in \partial Q: \lim _{y \rightarrow x, y \in Q} f(y)\right.$ does not exist $\}$. To prove that $\operatorname{dim} Z \leq d-2$, assume to the contrary that $Z$ contains a cell $W$ of dimension $d-1$. The boundary of the graph of $f$ has dimension smaller than $d$, so the set of points in the closure of $Q$ for which the fiber of the closure of the graph is infinite (i.e. the function has infinitely many accumulation points) has dimension smaller than $d-1$.

Using the cell decomposition we may assume that at any $x \in Z$ the function $f$ has finitely many accumulation points and that the definable functions $\limsup _{y \rightarrow x, y \in Q} f(y)$ and $\liminf _{y \rightarrow x, y \in Q} f(y)$ are continuous on $Z$. Fix $x_{0} \in Z$ and set $a=\limsup _{y \rightarrow x_{0}, y \in Q} f(y), b=\liminf _{y \rightarrow x_{0}, y \in Q} f(y)$. There exist numbers $c \in(a, b)$ and $e>0$ such that $|f(y)-c|>e$ in a neighborhood of $x_{0}$ in $\bar{Q}$. Let $Q_{1} \subset Q$ be a cell such that $\bar{Q}_{1}$ is a neighborhood of $x_{0}$ in $\bar{Q}$ and $|f(x)-c|>e$ on $Q_{1}$. This contradicts the connectedness of $Q_{1}$.

If

$$
Q=\left\{\left(x, x_{n}\right) \in \mathbb{R}^{n-1} \times \mathbb{R}: x \in Q_{1}, f(x)<x_{n}<g(x)\right\}
$$

is a cell of band type then

$$
\bar{Q}=Q \cup \operatorname{graph} f \cup \operatorname{graph} g \cup\left(\bar{Q} \cap\left(\partial Q_{1} \times \mathbb{R}\right)\right) .
$$


The cell $Q$ is "bounded from below and above" by cells of graph type, graph $g$ and graph $f$, which we shall call the top and bottom decks of $Q$. The closure $\bar{Q}$ of $Q$ is bounded by the closures of the top and bottom decks. In the case of cells of band type with only one deck or without a deck the closure is described similarly.

Lemma 8. Under the assumptions of Theorem 1 , for any $\varepsilon>0$ there exists a cell decomposition $\mathcal{T}$ of $\mathbb{R}^{k} \times \mathbb{R}^{n}$ compatible with $A_{1}, \ldots, A_{s}$ and satisfying the following conditions:

(1) for any cell $Q \in \mathcal{T}$ such that $\operatorname{dim} Q_{y}=n-1$ and any points $\left(x^{\prime}, y^{\prime}\right),\left(x^{\prime \prime}, y^{\prime \prime}\right) \in Q$ we have

$$
\delta\left(T_{x^{\prime}} Q_{y^{\prime}}, T_{x^{\prime \prime}} Q_{y^{\prime \prime}}\right)<\varepsilon
$$

(2) for any cell $Q \in \mathcal{T}$ such that $\operatorname{dim} Q_{y}=n$ for some $y \in \pi Q$ there exist cells $B_{1}, \ldots, B_{p} \in \mathcal{T}(p \leq 2 n)$ such that $\operatorname{dim}\left(B_{i}\right)_{y}=n-1$, $\left(B_{i}\right)_{y} \subset \bar{Q}_{y} \backslash Q_{y}$ and the set $\partial Q_{y} \backslash \bigcup\left(B_{i}\right)_{y}$ is a finite union of cells of dimension $\leq n-2$.

Proof. We use induction on $n$.

There exists a cell decomposition of $\mathbb{R}^{k} \times \mathbb{R}^{n}$ compatible with $A_{1}, \ldots, A_{s}$ and such that the corresponding decomposition of $\mathbb{R}^{k} \times \mathbb{R}^{n-1}$ satisfies the assertion of the lemma. Consequently, condition (1) holds for any cell of band type. For any cell $Q \subset \mathbb{R}^{k} \times \mathbb{R}^{n}$ such that $\operatorname{dim} Q_{y}=n-1$ and $Q_{y}$ is of graph type consider the map

$$
Q \ni(x, y) \mapsto T_{x} Q_{y} \in \mathbb{G}(n-1, n) .
$$

Since this map is definable we can assume, after refining the decomposition in $\mathbb{R}^{k} \times \mathbb{R}^{n-1}$, that condition (1) holds for any cell $Q$ such that $\operatorname{dim} Q_{y}=n-1$ and $Q_{y}$ is of graph type.

Fix a cell $Q$ such that $Q_{y}$ is an open cell. Clearly $Q$ is of band type, so

$$
Q=\left\{(y, x) \in Q_{1} \times \mathbb{R}: f\left(y, x_{1}, \ldots, x_{n-1}\right)<x_{n}<g\left(y, x_{1}, \ldots, x_{n-1}\right)\right\}
$$

where $Q_{1}$ is the projection of $Q$ onto $\mathbb{R}^{k} \times \mathbb{R}^{n-1}$.

By Lemma 7 there exists a definable subset $Z \subset \partial Q_{1}$ such that for any $y$ we have $\operatorname{dim} Z_{y}<n-2$ and the functions $f(y, \cdot)$ and $g(y, \cdot)$ extend continuously to $\left(\partial \bar{Q}_{1} \backslash Z\right)_{y}$. After refining we may assume that the decomposition of $\mathbb{R}^{k} \times \mathbb{R}^{n-1}$ is compatible with $Q_{1}, \bar{Q}_{1}$ and $Z$ and satisfies the assertions of the lemma.

We have constructed a cell decomposition of $\mathbb{R}^{k} \times \mathbb{R}^{n}$ such that

- for any cell $Q$ of this decomposition such that $Q_{y}$ is of graph type and $\operatorname{dim} Q_{y}=n-1$ condition (1) holds,

- for any cell $Q$ such that $Q_{y}$ is open, $Q=\left\{(y, x) \in Q_{1} \times \mathbb{R}: f\left(y, x_{1}, \ldots, x_{n-1}\right)<x_{n}<g\left(y, x_{1}, \ldots, x_{n-1}\right)\right\}$, 
there exist definable cells $\widetilde{B}_{1}, \ldots, \widetilde{B}_{p}$ such that $\left(\widetilde{B}_{i}\right)_{y} \subset \partial\left(Q_{1}\right)_{y}$ and $\left(\partial Q_{1}\right)_{y} \backslash \bigcup\left(\widetilde{B}_{i}\right)_{y}$ is a union of cells of dimension $<n-2$ and the functions $f(y, \cdot), g(y, \cdot)$ have continuous extensions $\widetilde{f}(y, \cdot), \widetilde{g}(y, \cdot)$ onto $\left(Q_{1}\right)_{y} \cup \cup\left(\widetilde{B}_{i}\right)_{y}$.

Put

$$
\begin{aligned}
B_{i}=\left\{(y, x) \in \mathbb{R}^{k} \times \mathbb{R}^{n}:\right. & \left(y, x_{1}, \ldots, x_{n-1}\right) \in \widetilde{B}_{i}, \\
& \left.\widetilde{f}\left(y, x_{1}, \ldots, x_{n-1}\right)<x_{n}<\widetilde{g}\left(y, x_{1}, \ldots, x_{n-1}\right)\right\}
\end{aligned}
$$

for $i=1, \ldots, p$, and

$$
B_{p+1}=\operatorname{graph} f, \quad B_{p+2}=\operatorname{graph} g .
$$

Clearly $\left(B_{1}\right)_{y}, \ldots,\left(B_{p+2}\right)_{y}$ are cells of dimension $n-1$, and $p+2 \leq 2 n$. We now show that $\operatorname{dim}\left(\partial Q_{y} \backslash \bigcup\left(\widetilde{B}_{i}\right)_{y}\right)<n-1$. Assume that $\partial Q_{y} \backslash \bigcup\left(\widetilde{B}_{i}\right)_{y}$ contains a cell $C$ of dimension $n-1$; we can assume (after refining the decomposition) that $C$ is a cell of the decomposition. If $C \subset \overline{\left(B_{p+1}\right)_{y}} \cup$ $\overline{\left(B_{p+2}\right)_{y}}$ then by Lemma 7 we would get $C \subset\left(B_{p+1}\right)_{y}$ or $C \subset\left(B_{p+2}\right)_{y}$, contrary to our assumptions. Consequently, $\left.C \cap \overline{\left(B_{p+1}\right)_{y}} \cup \overline{\left(B_{p+2}\right)_{y}}\right)=\emptyset$. This means that the projection of $C$ onto $\mathbb{R}^{n-1}$ is contained in one of the sets $\left(\widetilde{B}_{i}\right)_{y}, i=1, \ldots, p$. But then $C \subset\left(B_{i}\right)_{y} \cup \operatorname{graph} \widetilde{g}\left|\left(\widetilde{B}_{i}\right)_{y} \cup \operatorname{graph} \widetilde{f}\right|\left(\widetilde{B}_{i}\right)_{y}$, which contradicts the choice of $C$.

Lemma 9. Let $A \subset \mathbb{R}^{k} \times \mathbb{R}^{n}$ be a definable set and let $d:=\max \operatorname{dim} A_{y}$. For any $\varepsilon>0$ there exists a cell decomposition $\mathcal{T}$ of $\mathbb{R}^{k} \times \mathbb{R}^{n}$ compatible with $A$ such that for any cell $Q$ of $\mathcal{T}$ satisfying $\operatorname{dim} Q_{y}=d$ and any points $\left(x^{\prime}, y^{\prime}\right),\left(x^{\prime \prime}, y^{\prime \prime}\right) \in Q$ we have

$$
\delta\left(T_{x^{\prime}} Q_{y^{\prime}}, T_{x^{\prime \prime}} Q_{y^{\prime \prime}}\right)<\varepsilon .
$$

Proof. The proof is similar to the proof of (1) in Lemma 8.

5. Proof of Theorem 1. We shall prove the theorem using induction on $n$. Since in $\mathbb{R}$ every cell is a point, segment, half-line or line, the theorem is obvious for $n=1$.

We shall construct a sequence $\mathcal{T}_{i}$ of definable stratifications compatible with sets $A_{1}, \ldots, A_{s}$ and such that for each stratum $Q \in \mathcal{T}_{i}$ with $\operatorname{dim} Q>$ $n+k-i$ and any point $y \in \pi(Q)$ the fiber $Q_{y}$ is a cell satisfying the Whitney property with exponent 1 . We can take as $\mathcal{T}_{0}$ any definable stratification compatible with $A_{1}, \ldots, A_{s}$. Then $\mathcal{T}_{i+1}$ is constructed by refinement of strata from $\mathcal{T}_{i}$ of dimension at most $n+k-i$.

Using Lemmata 8 and 9 it is enough to prove that for any cell $Q$ satisfying the assertions of the lemma there are subsets $Q_{1}, \ldots, Q_{r} \subset Q$ such that $\operatorname{dim}\left(Q \backslash \bigcup_{i} Q_{i}\right)<\operatorname{dim} Q$ and for any point $y \in \pi\left(Q_{i}\right)$ the fiber $\left(Q_{i}\right)_{y}$ is a cell satisfying the Whitney property with exponent 1. 
CASE I. If $\operatorname{dim} Q_{y}=n$ (i.e. $Q_{y}$ is an open cell in $\mathbb{R}^{n}$ ) then there exist cells $B_{1}, \ldots, B_{p}(p \leq 2 n)$ such that

- for any points $\left(x^{\prime}, y^{\prime}\right),\left(x^{\prime \prime}, y^{\prime \prime}\right) \in B_{i}$ we have

- $\operatorname{dim}\left(B_{i}\right)_{y}=n-1$,

$$
\delta\left(T_{x^{\prime}}\left(B_{i}\right)_{y^{\prime}}, T_{x^{\prime \prime}}\left(B_{i}\right)_{y^{\prime \prime}}\right)<\varepsilon,
$$

- $\partial Q_{y} \backslash \bigcup\left(B_{i}\right)_{y}$ is a finite sum of cells of dimension $\leq n-2$.

By Lemma 5 there exists a line $L$ in $\mathbb{R}^{n}$ such that for any point $(x, y) \in Q$ we have $\delta\left(L, T_{x}\left(B_{i}\right)_{y}\right)>\alpha$, where $i=1, \ldots, p$, and $\alpha$ is a constant depending only on $n$. Changing coordinates in $\mathbb{R}^{n}$ we can assume that $L$ is the $x_{n}$-axis. Every cell $\left(B_{i}\right)_{y}$ is locally the graph of a definable function with derivative bounded by a constant $M_{n}$ depending only on $n$.

Using cell decomposition and the inductive hypothesis we get a cell decomposition $\mathcal{C}$ of $\mathbb{R}^{k} \times \mathbb{R}^{n}$ compatible with $Q$ and $B_{i}$ such that the induced decomposition $\mathcal{C}_{1}$ of $\mathbb{R}^{k} \times \mathbb{R}^{n-1}$ satisfies the assertion of the theorem. Let $\widetilde{\mathcal{C}}$ be a cell decomposition of $\mathbb{R}^{k} \times \mathbb{R}^{n}$ given by the cell decomposition $\mathcal{C}_{1}$ of $\mathbb{R}^{k} \times \mathbb{R}^{n-1}$ and the sets $B_{i}$ (this means that for any cell of $\widetilde{\mathcal{C}}$ its projection is an element of $\mathcal{C}_{1}$, and each cell of graph type is a subset of some $B_{i}$ ).

For any cell $K \in \widetilde{\mathcal{C}}$ of graph type such that $\operatorname{dim} K_{y}=n-1$ we have $K_{y} \subset\left(B_{i}\right)_{y}$ for some $i$, and so $K_{y}$ is the graph of a function with derivative bounded by the constant $M_{n}$ and defined on some cell in $\mathbb{R}^{n-1}$ satisfying the Whitney property with exponent 1 and coefficient $L_{n}:=L_{n-1} \sqrt{1+M_{n-1}^{2}}$ depending only on $n$.

Consequently, each cell $K \in \widetilde{\mathcal{C}}$ satisfies the Whitney property with exponent 1 and coefficient depending only on $n$ because its projection and decks satisfy the Whitney property.

Let $Q_{1}, \ldots, Q_{r}$ be cells of the decomposition $\widetilde{\mathcal{C}}$ such that $\operatorname{dim}\left(Q_{i}\right)_{y}=n$ and $Q_{i} \cap Q \neq \emptyset$. Clearly $\operatorname{dim}\left(Q_{y} \backslash \bigcup_{i}\left(Q_{i}\right)_{y}\right) \leq n-1$ and $\left(Q_{i}\right)_{y}$ satisfies the Whitney property with exponent 1 and coefficient depending only on $n$. Since $\partial Q_{y} \backslash \bigcup\left(B_{j}\right)_{y}$ is a finite sum of cells of dimension $\leq n-2$ and $Q_{i} \cap B_{j}=\emptyset$ we get $Q_{i} \subset Q$.

CASE II. If $d=\operatorname{dim} Q_{y}<n$ then there exists a line $L$ in $\mathbb{R}^{n}$ such that $\delta\left(L, T_{x} Q_{y}\right) \geq 1-\varepsilon$ for any $(x, y) \in Q$. After a change of variables in $\mathbb{R}^{n}$ we can assume that $L=\left(x_{1}=\cdots=x_{n-1}=0\right)$. Then every $Q_{y}$ is the graph of a $\mathcal{C}^{1}$ function with derivative bounded by an arbitrarily small positive constant (depending on $\varepsilon$ ) defined on the set $\widetilde{Q}_{y}$, where $\widetilde{Q}$ is the projection of $Q$ onto $\mathbb{R}^{k} \times \mathbb{R}^{n-1}$.

Applying the inductive hypothesis we can find $\widetilde{Q}_{1}, \ldots, \widetilde{Q}_{r} \subset \widetilde{Q}$ which are definable cells satisfying the Whitney property with exponent 1 and coefficient depending only on $d$, and such that $\operatorname{dim}\left(\widetilde{Q} \backslash \bigcup_{i} \widetilde{Q}_{i}\right)<\operatorname{dim} \widetilde{Q}$. Now, put $Q_{i}=Q \cap\left(\widetilde{Q}_{i} \times \mathbb{R}\right)$. 
Acknowledgements. The author would like to thank W. Pawłucki for suggesting the problem and for his help during the preparation of this paper.

\section{References}

[1] L. van den Dries, Tame Topology and o-Minimal Structures, London Math. Soc. Lecture Note Ser. 248, Cambridge Univ. Press, 1998.

[2] L. van den Dries and C. Miller, Extending Tamm's theorem, Ann. Inst. Fourier (Grenoble) 44 (1994), 1367-1395.

[3] R. M. Hardt, Some analytic bounds for subanalytic sets, in: Differential Geometric Control Theory, Progr. Math. 27, Birkhaüser, 1983, 259-267.

[4] K. Kurdyka, On a subanalytic stratification satisfying a Whitney property with exponent 1, in: Real Algebraic Geometry (La Tourballe, 1991), Lecture Notes in Math. 1524, Springer, 1992, 316-322.

[5] J. Stasica, The Whitney condition for subanalytic sets, Zeszyty Nauk. Uniw. Jagiell. Prace Mat. 23 (1982), 211-221.

Institute of Mathematics

Cracow University of Technology

Warszawska 24

31-155 Kraków, Poland

E-mail: bkocel@pk.edu.pl

Received 19.12.2006

and in final form 30.4.2007 